\title{
Carbon-covered magnetic nanomaterials and their application for the thermolysis of cancer cells
}

This article was published in the following Dove Press journal:

International Journal of Nanomedicine

10 March 2010

Number of times this article has been viewed

\section{Yang $\mathrm{Xu}$ \\ Meena Mahmood' \\ Ashley Fejleh' \\ Zhongrui $\mathrm{Li}^{\prime}$ \\ Fumiya Watanabe' \\ Steve Trigwell ${ }^{2}$ \\ Reginald B Little ${ }^{3}$ \\ Vasyl P Kunets ${ }^{4}$ \\ Enkeleda Dervishi' \\ Alexandru R Biris ${ }^{5}$ \\ Gregory J Salamo ${ }^{4}$ \\ Alexandru S Biris'}

'Nanotechnology Center and Applied Science Department, University of Arkansas at Little Rock, Little Rock, AR, USA; ${ }^{2}$ Applied Science and Technology, ASRC Aerospace, NASA Kennedy Space Center, FL, USA; ${ }^{3}$ Department of Chemistry, Elizabeth City State University, Elizabeth City, NC, USA; ${ }^{4}$ Physics Department, University of Arkansas, Fayetteville, AR, USA; ${ }^{5}$ National Institute for Research and Development of Isotopic and Molecular Technologies, Cluj Napoca, Romania
Correspondence: Yang Xu and Alexandru $S$ Biris

Nanotechnology Center and Applied Science Department, University of Arkansas at Little Rock, Little Rock, AR 72204, USA

Tel +I 50 I 682 5I66; + I 50I 6837458

Fax $+|50| 683760 \mid$

Email yxxu@ualr.edu; asbiris@ualr.edu
Abstract: Three types of graphitic shelled-magnetic core ( $\mathrm{Fe}, \mathrm{Fe} / \mathrm{Co}$, and $\mathrm{Co}$ ) nanoparticles (named as $\mathrm{C}-\mathrm{Fe}, \mathrm{C}-\mathrm{Fe} / \mathrm{Co}$, and $\mathrm{C}$-Co NPs) were synthesized by radio frequency-catalytic chemical vapor deposition (RF-cCVD). X-ray diffraction and X-ray photoelectron spectroscopy analysis revealed that the cores inside the carbon shells of these NPs were preserved in their metallic states. Fluorescence microscopy images indicated effective penetrations of the NPs through the cellular membranes of cultured cancer HeLa cells, both inside the cytoplasm and the nucleus. Low RF radiation of $350 \mathrm{kHz}$ induced localized heating of the magnetic NPs, which triggered cell death. Apoptosis inducement was found to be dependent on the RF irradiation time and NP concentration. It was showed that the Fe-C NPs had a much higher ability of killing the cancer cells (over 99\%) compared with the other types of NPs (C-Co or $\mathrm{C}-\mathrm{Fe} / \mathrm{Co}$ ), even at a very low concentration of $0.83 \mu \mathrm{g} / \mathrm{mL}$. The localized heating of NPs inside the cancer cells comes from the hysteresis heating and resistive heating through eddy currents generated under the RF radiation. The RF thermal ablation properties of the magnetic NPs were correlated with the analysis provided by a superconducting quantum interference device (SQUID).

Keywords: graphitic shelled, magnetic nanoparticles, radio frequency, thermal ablation, HeLa cells

\section{Introduction}

Magnetic nanoparticles (NPs) present a great interest for a wide range of applications, such as medical diagnostics and therapeutics, ${ }^{1,2}$ magnetic resonance imaging (MRI) ${ }^{3,4}$ catalysis, ${ }^{5}$ data storage, ${ }^{6}$ and environmental remediation. ${ }^{7}$ The low stability of these NPs in severe environments presents a challenge, since it limits their usage without a protective shell. While many methods have been developed for the synthesis of magnetic NPs with different protective coatings, the successful use of such magnetic NPs in biomedical applications is highly dependent on their biostability and surface active properties. Magnetic NPs have a large magnetic moment and behave like giant paramagnetic atoms with fast response to applied magnetic fields with negligible remanence (residual magnetism) and coercivity (the field required to bring the magnetization to zero). Although most studies have focused on the development of polymer or silica protective coatings, carbon-protected magnetic NPs have recently received more attention ${ }^{8-10}$ due to the relatively higher chemical and thermal stability, inertness, as well as enhanced biocompatibility of carbon. ${ }^{11,12}$ Moreover, carbon-coated NPs can be preserved in the metallic state, and thus have a higher magnetic moment than their corresponding oxides. ${ }^{13}$ Core-shell nanostructures often 
exhibit improved physical and chemical properties over their single-component counterparts, which lends to a broader range of applications.

In this paper, $\mathrm{Fe}, \mathrm{Fe} / \mathrm{Co}$, and $\mathrm{Co}$ NPs covered with layers of graphitic carbon were respectively synthesized by radio frequency-catalytic chemical vapor deposition (RF-cCVD). ${ }^{10} \mathrm{C}-\mathrm{Fe}$ NPs showed the highest efficiency for inducing cell death compared to their $\mathrm{C}-\mathrm{Fe} / \mathrm{Co}$ and $\mathrm{C}-\mathrm{Co}$ counterparts. It was determined that the C-Fe NPs had a much higher ability to kill the cancer cells (over 99\%) compared with the other types of NPs, such as the C-Co $\mathrm{NPs},{ }^{10}$ even at much lower concentrations and for shorter $\mathrm{RF}$ radiation times.

The NPs were shown to act as strong RF absorbers and temperature inducers. NPs with various metallic cores induced the death of the HeLa cells during in vitro experiments due to their strong magnetic properties. The C-Co NPs exhibited superparamagnetism at room temperature, but at $5 \mathrm{~K}, \mathrm{C}-\mathrm{Fe}$ NPs demonstrated a higher coercivity. Carbon-coated magnetic NPs could be further developed into components of an efficient and reliable tumor therapy for the successful thermal ablation of both individual cancer cells and tumors. To achieve this goal, it is desired to synthesize magnetic NPs that can produce the required heat to kill cancer cells under RF radiation, but do so in low concentrations in order to avoid their possible undesired cytotoxic effects. This may be accomplished by improving the response of the NPs to RF radiation, as well as improving their surface properties of the NPs for specific cellular uptake.

\section{Methods}

\section{$\mathrm{C}-(\mathrm{Co}, \mathrm{Fe} / \mathrm{Co}, \mathrm{Fe}) \mathrm{NPs}$ preparation}

$\mathrm{C}-(\mathrm{Co}, \mathrm{Fe} / \mathrm{Co}, \mathrm{Fe}) \mathrm{NPs}$ were prepared using the RF-cCVD method as follows: For C-Fe/Co NPs, $1.00 \mathrm{~g}$ of nanopowder $\mathrm{MgO}$ (Sigma-Aldrich, St. Louis, MO) with $0.145 \mathrm{~g}$ of $\mathrm{Fe}\left(\mathrm{NO}_{3}\right)_{3} \cdot 9 \mathrm{H}_{2} \mathrm{O}$ and $0.105 \mathrm{~g}$ of $\mathrm{Co}\left(\mathrm{NO}_{3}\right)_{2} \cdot 6 \mathrm{H}_{2} \mathrm{O}$ were impregnated in $50 \mathrm{~mL}$ of ethanol and sonicated for one hour. For $\mathrm{C}-\mathrm{Fe}$ and C-Co NPs, $0.29 \mathrm{~g}$ of $\mathrm{Fe}\left(\mathrm{NO}_{3}\right)_{3} \cdot 9 \mathrm{H}_{2} \mathrm{O}$ and $0.21 \mathrm{~g}$ of $\mathrm{Co}\left(\mathrm{NO}_{3}\right)_{2} \cdot 6 \mathrm{H}_{2} \mathrm{O}$ were used instead, respectively. After drying at $100{ }^{\circ} \mathrm{C}$, the powder was ground finely and typically $0.40 \mathrm{~g}$ was used for methane-based cCVD in an RF generator ( $350 \mathrm{kHz}, 5 \mathrm{~kW}$ of RF induction). The samples were heated in a $\mathrm{H}_{2}$ atmosphere at $100 \mathrm{~mL} / \mathrm{min}$ flow rate up to $800{ }^{\circ} \mathrm{C}$ and then subjected to a methane flow of $400 \mathrm{~mL} / \mathrm{min}$ for five minutes. Finally, the samples were washed with concentrated $\mathrm{HCl}$ and collected using a $0.2 \mu \mathrm{m}$ filter (ISOPORE ${ }^{\mathrm{TM}}$; Millipore, Billerica, MA).

\section{Transmission electron microscopy analysis}

Transmission electron microscopy (TEM) images were collected on a JEM2100F TEM (JEOL Inc., Pleasanton, CA) with an acceleration voltage of $200 \mathrm{kV}$. For this analysis, NP powder was dispersed in 2-propanol and sonicated for 10 minutes. A few drops of the suspension were deposited on a TEM grid. This preparation was then dried and evacuated before analysis. Size distributions for each NP preparation were obtained from TEM analysis based on the measurement of 100 NPs.

\section{Atomic force microscopy analysis}

Atomic force microscopy (AFM) image was acquired with a Dimension 3100 instrument (Veeco, Plainview, NY) using tapping mode measurements. The cantilevers exhibited resonant frequencies around $300 \mathrm{kHz}$ and force constants of about $40 \mathrm{~N} / \mathrm{m}$. This instrument has a scan range of $90 \mu \mathrm{m}$ for the " $\mathrm{x} / \mathrm{y}$ " direction and $6 \mu \mathrm{m}$ for the " $\mathrm{z}$ " direction. The sample was prepared by dropping the NP isopropanol solution on Si substrates. Next, the substrates were air-dried and placed directly under the AFM tip for morphology analysis.

\section{X-ray photoelectron spectrometer analysis}

X-ray photoelectron spectrometer (XPS) measurements were performed using K-Alpha (Thermo Scientific, Waltham, MA) at a background pressure of $1 \times 10^{-9}$ torr and a monochromated $\mathrm{Al} K \alpha(\mathrm{hu}=1456.6 \mathrm{eV}) \mathrm{X}$-ray source and a combined lowenergy electron/ion flood gun for charge neutralization. The collected data were referenced to the graphite $\mathrm{C} 1 \mathrm{~s}$ peak to $284.5 \mathrm{eV}^{14}$ Detection limits for XPS were approximately 0.1 to 1.0 atomic $\%$ depending upon the sensitivity of the elements.

\section{Temperature-dependent measurements of magnetization}

These measurements were performed by using a MPMS- $5 \mathrm{~S}$ superconducting quantum interference device (SQUID) magnetometer from Quantum Design Inc (San Diego, CA). The maximum allowed magnetic field was 5 Tesla and the magnetic field sweeps could be performed for both polarities in a temperature range of $1.8-300 \mathrm{~K}$. The longitudinal magnetic moment was measured using the DC technique. Each point was taken as an average of four single measurements. Samples were mounted in gelatin capsules having a negligible magnetic response. The weight of synthesized 
materials was measured using a weight measurement station (XL-410; Denver Instruments, Denver, CO).

Three types of measurements were performed:

1. Zero-field-cooled (ZFC): samples were cooled down to temperature of $5 \mathrm{~K}$ with zero applied magnetic field, then a magnetic field of 30 Oersted (unit of magnetizing field and also known as magnetic field strength or intensity $\mathrm{Oe}$ ) was applied and temperature dependent measurements were taken during warm up.

2. Field-cooled (FC): samples were cooled down to $5 \mathrm{~K}$ at an applied magnetic field of 30 Oe and measurements were taken during warm up with the magnetic field held constant.

3. Hysteresis loops were measured for each of the samples at $300 \mathrm{~K}$ and $5 \mathrm{~K}$ in the range of -2 to 2 Tesla.

\section{HeLa cell culture and incubation with NPs}

For the cell culture, mammalian cervical cancer cells (HeLa cells) were seeded in $10 \mathrm{~cm}^{2}$ culture plates $\left(0.5 \times 10^{6}\right.$ cells $/$ plate $)$ with growth medium (minimum essential medium containing $10 \%$ fetal bovine serum and $1 \%$ penicillin 100 unit $/ \mathrm{mL}$, streptomycin $100 \mu \mathrm{g} / \mathrm{mL}$ ) and incubated in a humidified incubator $\left(37^{\circ} \mathrm{C}, 5 \% \mathrm{CO}_{2}\right)$. For the subculture, cells were dissociated by $1 \times$ trypsin/EDTA in PBS and counted and plated into $35 \mathrm{~mm}$ culture plates at a density of $5 \times 10^{4}$ cells/plate and supplemented with growth medium containing various concentrations of NPs $(0-3.33 \mu \mathrm{g} / \mathrm{mL})$. All the NPs were incubated with HeLa cells for 24 hours before RF treatment.

\section{Cellular death studies performed using ethidium bromide and acridine orange $(E B / A O)$ staining methods}

For $\mathrm{EB} / \mathrm{AO}$ staining, $25 \mu \mathrm{L}$ of the cell suspension $\left(5 \times 10^{4}\right.$ cells $\left./ \mu \mathrm{L}\right)$ was mixed with $2 \mu \mathrm{L}$ of the dye mixture, containing $0.15 \mathrm{mg} \mathrm{mL}^{-1}$ of acridine orange and $0.50 \mathrm{mg} \mathrm{mL}^{-1}$ of ethidium bromide in $1 \times$ phosphate-buffered saline (PBS). After staining, cells were then visualized by using a fluorescence microscope (Olympus, Center Valley, PA) at 10× and $40 \times$ objectives equipped with a digital camera. Photographs were taken using randomly selected fields of view. To determine the percentage of cells undergoing death, photographs taken were used for counting the number of live (green) and dead (orange) cells. Acridine orange stains live cells green whereas ethidium bromide stains dead cells as red. Approximately 200-300 cells per treatment were counted to obtain statistical cell death information.

\section{RF heating and determination on surface temperature of NP powder}

Cells were subjected to RF induction at $350 \mathrm{kHz}$ and $5 \mathrm{~kW}$ for time periods ranging from 2 to 45 minutes. The calculation of the cells was done under fluorescence microscopy counting the dead and alive cells. NP powders were placed in the Petri dish individually and subject to RF heating for five minutes. Before, during, and after RF heating, an infrared thermometer (PTM 01, Russia) was used to check the temperature of the powdered samples. All heating studies were repeated at least five times in all conditions.

\section{Results and discussions}

The magnetic NPs were synthesized by a RF-cCVD process. ${ }^{10}$ AFM and TEM analysis revealed that the average size of the $\mathrm{C}-\mathrm{Fe}, \mathrm{C}-\mathrm{Fe} / \mathrm{Co}$ and C-Co NPs was $8 \pm 1.0 \mathrm{~nm}, 9 \pm 1.5 \mathrm{~nm}$ and $7 \pm 1.2 \mathrm{~nm}$, respectively, with the mean and standard deviation of sizes measured by TEM for $\sim 100$ NPs in each samples. The NPs were covered with 5-8 layers, 7-10 layers, and 2-4 layers of graphitic carbon respectively, as shown in Figure 1.

XPS analysis was carried out to inspect the oxidation states of the encapsulated NPs. Core level XPS profiles of the $\mathrm{C} 1 \mathrm{~s}, \mathrm{O} 1 \mathrm{~s}, \mathrm{Fe} 2 \mathrm{p}$, and $\mathrm{Co} 2 \mathrm{p}$ were taken at a pass energy of $50 \mathrm{eV}$ in $0.01 \mathrm{eV}$ steps for higher resolution. The Co2p and $\mathrm{Fe} 2 \mathrm{p}$ peaks were located at $778.6( \pm 0.1) \mathrm{eV}$ and 707.1 $( \pm 0.1) \mathrm{eV}$ (Figures $2 \mathrm{a}$ and $2 \mathrm{~b}$, respectively), indicating the metallic state (Co oxides $\sim 780-781 \mathrm{eV}$, Fe oxides $709-711 \mathrm{eV}) .{ }^{15}$ This was also confirmed by the O1s peak at $532.7 \mathrm{eV}$, since the $\mathrm{O} 1 \mathrm{~s}$ peak for $\mathrm{Co}$-oxide is $532.35 \mathrm{eV} .^{16}$ Therefore, the metallic states of the Fe, Fe/Co, and Co NPs were well protected by the graphite shells.

In order to confirm the biocompatibility of the different types of NPs, cytotoxicity studies involving HeLa Cells were carried out whereby the cells were cultured with the C-Fe, $\mathrm{C}-\mathrm{Fe} / \mathrm{Co}$, and C-Co NPs and these cultures were analyzed for cell viability. The results indicated that most (99.4 to $99.7 \%$ ) of the total cultured cells were alive, revealing a low level of toxicity of these magnetic NPs. Furthermore, it was found that $\mathrm{RF}$ radiation alone hardly induced any effect to the HeLa cells since around $93.0 \%$ of the cells still survived after RF treatment depending on the radiation time.

HeLa Cells cultured with various concentrations of magnetic NPs were introduced inside a water-cooled coil coupled to a radiofrequency generator with the frequency of $350 \mathrm{kHz}$, which is far lower than the $10 \mathrm{MHz}$ to $300 \mathrm{GHz}$ that is commonly used. ${ }^{17}$ The depth of penetration 
A

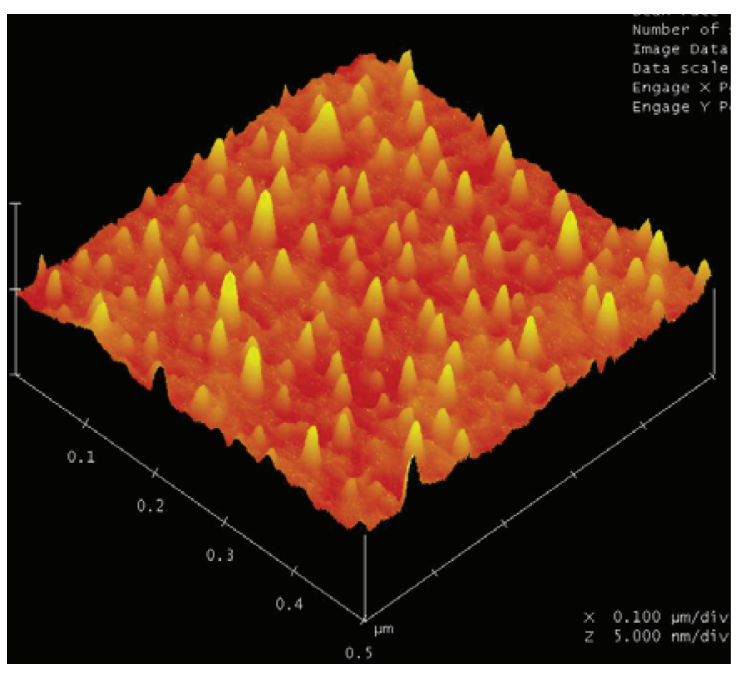

B

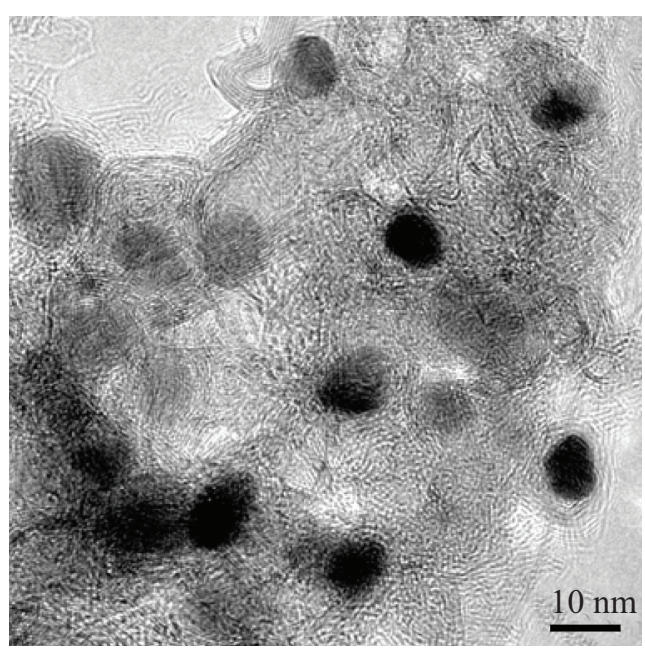

C

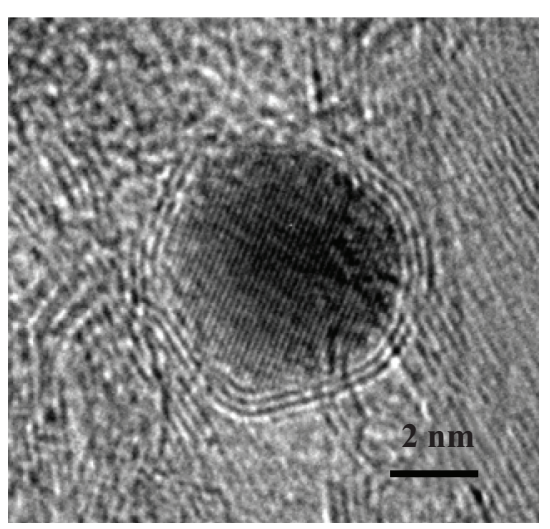

D

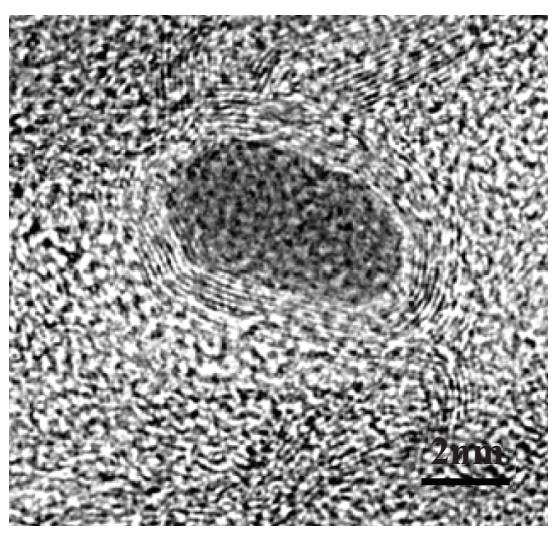

E

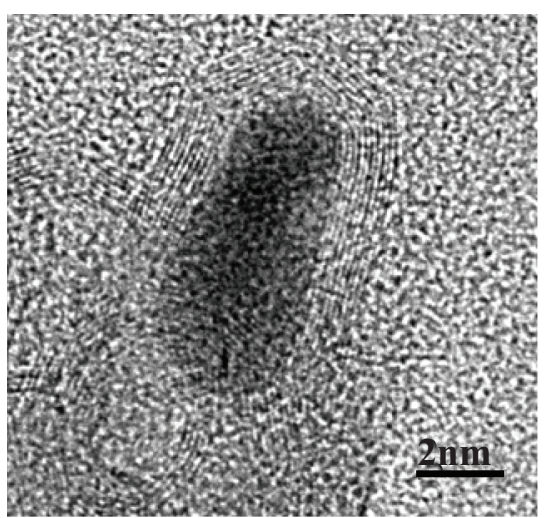

Figure I A) AFM topographic images showing individual C-Co NPs. B) Low and C, D, E) high magnification TEM images of graphitic C) C-Co, D) C-Fe and E) C-Fe/Co NPs obtained by RF-cCVD method.

Abbreviations:

of $\mathrm{RF}$ radiation in human tissue decreases as the frequency increases. Current clinical microwave radiometers operate in the $\mathrm{GHz}$ frequency range. The effective depth of the RF penetration drops from $17 \mathrm{~cm}$ at $85 \mathrm{MHz}$ to $7 \mathrm{~cm}$ at $220 \mathrm{MHz}$. Thus, RF below $200 \mathrm{MHz}$ are more desirable for the thermal ablation of cells or tissues located 1-20 cm deep into the body. Such low frequency radiation has the ability to penetrate the biological tissues efficiently and present a path for cancer treatment deep inside the body (for example, at $400 \mathrm{kHz}$, field penetration into $15 \mathrm{~cm}$ of tissue is $>99 \%) .{ }^{18}$ At frequencies below $100 \mathrm{MHz}$, the RF power deposition in patients is more evenly distributed over the exposed body volume than currently assumed. ${ }^{19}$ The frequency of the AC electromagnetic field should be chosen based on a compromise; namely, it must be higher than the frequency capable of provoking neuromuscular response and lower than the frequencies causing overheating of healthy tissues. It is believed that the frequency should be in the range $100-1000 \mathrm{kHz}$; in this case, correctly chosen frequencies and electromagnetic field strengths produce no notable side effects of the AC magnetic field in vivo. ${ }^{20}$ The schematic diagram of this setup was shown in our previous work. ${ }^{10}$ After the RF irradiation, the total number of live and dead cells was immediately counted through visualization by fluorescence microscopy. Figure $3 \mathrm{a}$ shows the results of the influence of the RF exposure for time periods of 2 to 30 minutes on the induction of apoptosis in the cells. If the concentration of the NPs was kept constant, the number of dead cells gradually increased after extending the RF-heating time. The three types of NPs were kept at the same low concentration (about $0.83 \mu \mathrm{g} / \mathrm{mL}$ ) and 


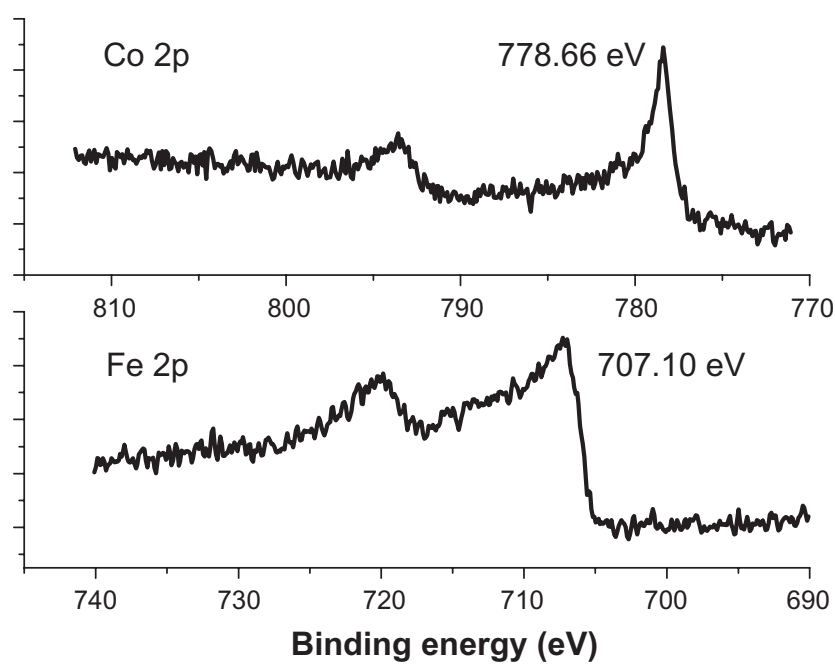

Figure 2 XPS spectra of the Co2p and Fe2p peaks represent the patterns of C-Fe and C-Co magnetic NPs.

Abbreviations:

were incubated with the HeLa Cells. After the RF heating, C-Fe NPs exhibited the highest killing rate of the HeLa Cells, as presented in Figure 3a. During only two minutes of RF exposure, $67.0 \%$ of cells were observed to have died. Increasing the heating time to 30 minutes caused the percentage of dead cells to reach about $74.5 \%$, which is 2.14 times and 2.69 times higher than that induced by the $\mathrm{C}-\mathrm{Fe} / \mathrm{Co}$ NPs and C-Fe NPs, respectively. The results indicated that the C-Fe NPs were the best RF absorbers and induced cellular death in the shortest time and at the lowest concentrations. Moreover, for RF exposure times longer than 10 minutes, the percentage of dead cells increased rather slowly (around $10 \%$ or less) depending on the type of NPs used in the experiment. As a result, if the exposure time was kept constant, the percentage of dead cells was found to be highly dependent on the NP concentration. As shown in Figure 3b, when the NP concentration was increased to $3.33 \mu \mathrm{g} / \mathrm{mL}$, C-Fe NPs killed almost $98.88 \%-100 \%$ of the exposed cells with only two minutes of RF exposure. However, for the C-Co NPs, a significantly higher concentration $(\sim 20 \mu \mathrm{g} / \mathrm{mL})$ was required to cause the same effect. ${ }^{10}$

It is believed that there will be variations in the amount and the numbers of NPs that will penetrate each individual cell. Although these variations exist, over a certain period of time, all cells will be heated up even at lower concentrations of NPs absorbed. The heat from one cell will propagate to the adjacent ones and therefore create a uniform temperature distribution over a certain area. This process of heat transfer is believed to enhance the overall thermodynamic process used to destroy the cells and the tumors. It is thought that cell death occurs not only from the NPs that are absorbed into the cells but also from the NPs attached to the cells and the extracellular medium.

Temperature changes of the NP powder upon exposure to $\mathrm{RF}$ radiation were studied in order to investigate the heating effects generated by the three different magnetic NPs. Five minutes of RF heating was considered sufficient in order to highlight the heating rates and the temperature differences between the three types of NPs. The high sensitivity thermal analysis indicated that the RF induced temperatures, as well as their heating rates, rose and were found to be dependent mostly upon the mass of the NPs used for the measurements. The C-Fe NPs were shown to be the best hyperthermia agents for killing the cancer cells since they were found to reach temperatures higher than the $\mathrm{C}-\mathrm{Fe} / \mathrm{Co}$ and $\mathrm{C}-\mathrm{Co} \mathrm{NPs}$ under identical RF exposure conditions (Figure 3c). Based on these experimental results and given the significant mass difference between the NPs present inside the cells and the cells themselves, the death of the cells is not expected to occur as a result of the bulk heating of the entire cell structures, but rather due to the localized damages of the cell such as nuclear membrane destruction, DNA fragmentation, and thermal denaturation of proteins (which happen at temperatures higher than $55^{\circ} \mathrm{C}$ ). As shown in Figure 4, after the NPs were taken up into the HeLa cell cytoplasm they were able to cross the various intercellular membranes and reach the nucleus. ${ }^{10}$ Due to the localized RF heating provided by the NPs, the cells were found to go through an apoptotic process, and subsequent cellular decomposition (one of the signs of the apoptotic process: the cell membrane blebbing) was observed as a result (Figure 4c). Compared to different NPs, as shown in Figures $4 \mathrm{~d}-\mathrm{f}$, the $\mathrm{C}$-Fe NPs revealed the highest efficiency for inducing cell death when compared to the C-Fe/Co NPs and C-Co NPs after two minutes of RF exposure. The disintegration of localized cellular environments such as nucleus, nuclear membranes, and DNA were believed to be the effective response resulting from RF-heat inducement into the NPs.

Magnetism of biological objects is negligibly small, so biologically compatible nontoxic magnetic NPs are used to strengthen the influence of an external magnetic field. Carbonshelled magnetic NP heating happens under RF radiation and it is possible that the NPs undergo an "auto-heating" event whereby the NPs generate their own heat energy based on molecular rotation. After heating for 60 to 90 seconds, the NP powders were found to reach the heating equilibrium (as shown in the insert in Figure 3c). This relaxation corresponds either to the physical rotation of particles directly inside the 
A

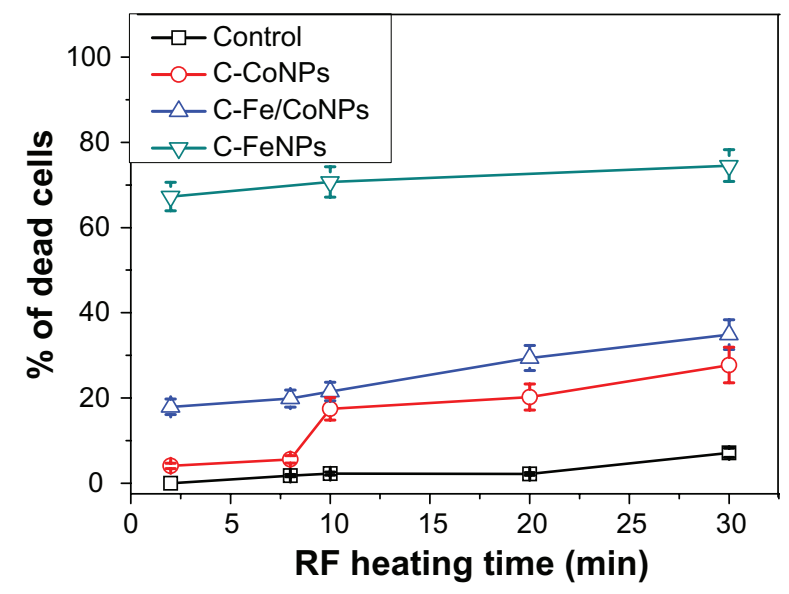

B

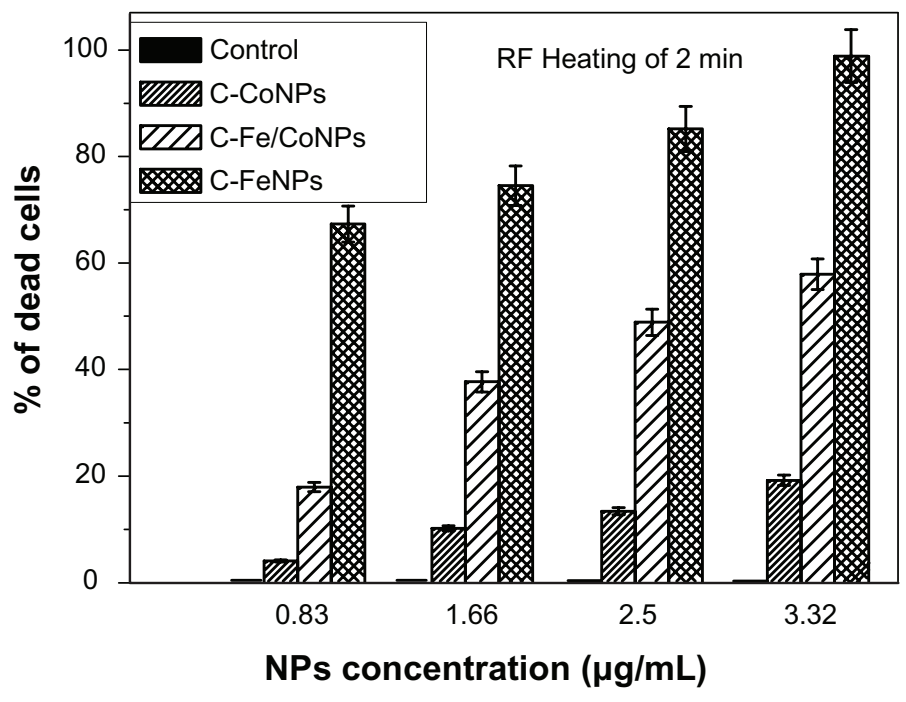

C

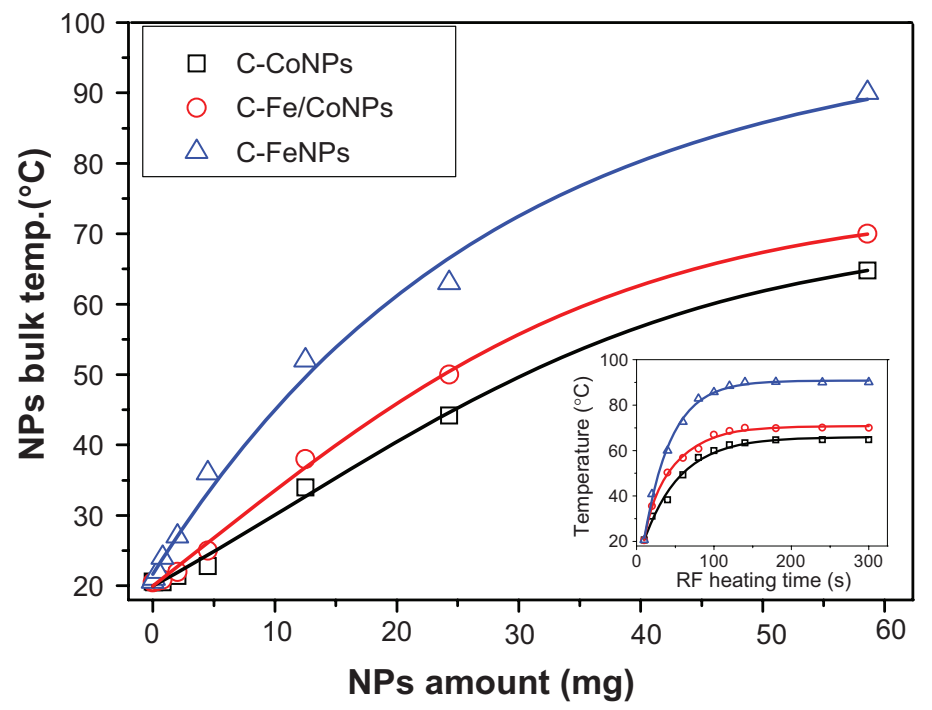

Figure 3 A) Cytotoxicity effects of the low concentration $(0.83 \mu \mathrm{g} / \mathrm{mL}) \mathrm{C}-\mathrm{Co}, \mathrm{C}-\mathrm{Fe} / \mathrm{Co}$, and C-Fe NPs on the HeLa cancer cells after 2 to 30 minutes of RF exposure. B) Effect of different concentrations of the C-Fe, C-Fe/Co, and C-Co NPs on the HeLa cells that died from $350 \mathrm{kHz}$ RF heating after two minutes of exposure time. C) Comparative RF-induced temperature variations as the function of different $\mathrm{C}-\mathrm{Fe}, \mathrm{C}-\mathrm{Fe} / \mathrm{Co}$, and $\mathrm{C}$-Co NP concentrations. Insert figure shows the temperature-rising characteristics of different magnetic NPs with the same amount under $350 \mathrm{kHz}$ RF exposure. 
A

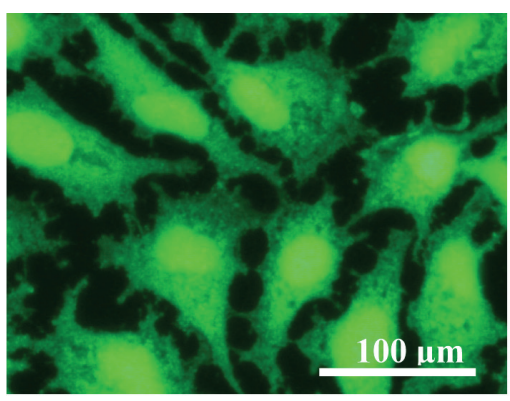

D

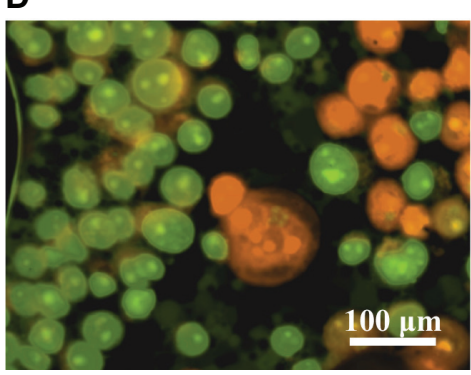

B

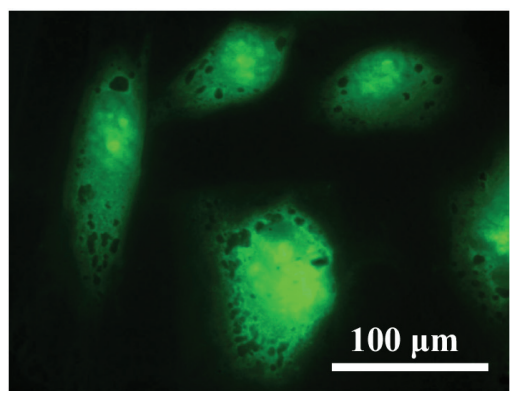

E

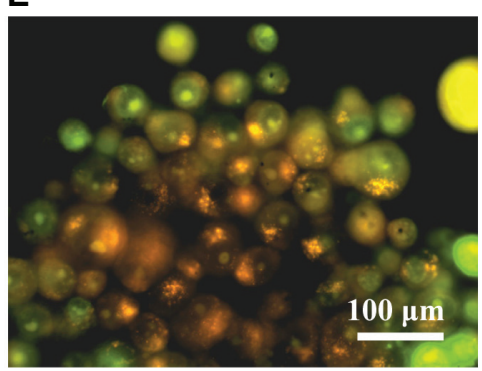

C

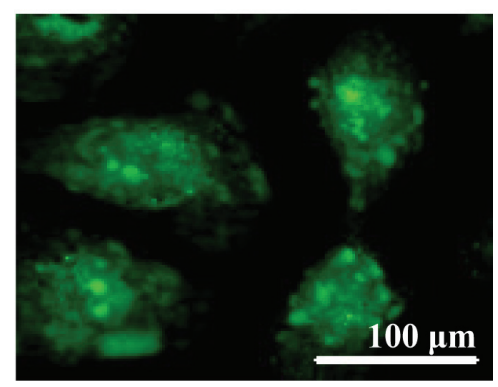

$\mathbf{F}$

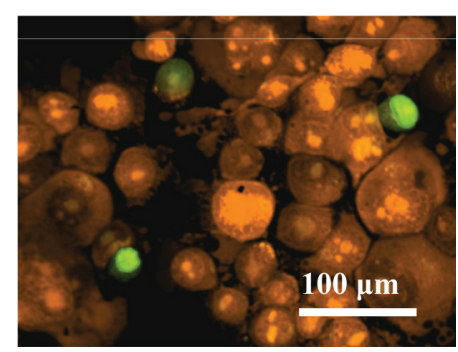

Figure 4 A) Normal HeLa cells without incubation of magnetic NPs; B) After 24 hours incubation time, the magnetic C-Co NPs were found to aggregate around and further penetrate into the nucleus of HeLa cells. C) The images took from the Olympus confocal microscopy indicating the membrane blebbing of HeLa cells incubated with magnetic C-Fe NPs after $350 \mathrm{kHz}$ RF heating for two minutes. Confocal microscopy images indicating the extensive live and dead cells after being incubated with the C-Co NPs D) C-Fe/Co NPs E), and C-Fe NPs F) after exposure to RF radiation for two minutes. The cells were stained in order to distinguish between the live (green for acridine orange) and the dead cells (orange for ethidium bromide).

liquid or to the rotation of nuclear magnetic moments inside each particle. Rotation of particles is usually referred to as Brownian rotation, and rotation of the magnetic moment inside each particle is called Néel relaxation. This "auto-heating" was attributed to the Néel relaxation ${ }^{21}$ of the spin of the NPs under low frequency $(350 \mathrm{kHz}) \mathrm{RF}$ radiation. The Néel relaxation time, $T_{N}$, of the magnetic NPs under an external magnetic field is expressed by following formula ${ }^{22}$ :

$$
T_{N}=\frac{\sqrt{\pi}}{2} T_{0} \frac{\operatorname{Exp}\left(K V_{m} / k_{B} T\right)}{\left(K V_{m} / k_{B} T\right)^{1 / 2}}
$$

where $T_{0}$ is the relaxation time constant and has the order of $10^{-9} \mathrm{~s}, K$ is the anisotropy constant, and $V_{M}$ is the magnetic volume of particles. The Néel relaxation time, $T_{N}$, was determined as the ratio of the energy of magnetic anisotropy of superparamagnetic particles to the thermal energy. The temperature of the three NPs increased rapidly in the initial stage (from 0-60 s) which can be seen from the curves in Figure $3 \mathrm{c}$ inset. After about 200 seconds, the final temperatures of $65^{\circ} \mathrm{C}, 70^{\circ} \mathrm{C}$, and $90^{\circ} \mathrm{C}$ were reached for $\mathrm{Co}, \mathrm{Fe} / \mathrm{Co}$, and Co NPs, respectively. This is due to the thermally stable spin rotation of the ferromagnetic NPs caused by the RF induced hysteresis loss. These spin properties are attributed to the particle size for the $\mathrm{C}-\mathrm{Fe}, \mathrm{C}-\mathrm{Fe} / \mathrm{Co}$, or C-Co NPs with diameters $<10 \mathrm{~nm}$ as shown in Figure $5 \mathrm{a}$.
In the RF-induced localized heating, there are two major heating mechanisms in the magnetic NPs. (1) Eddy currents: ferromagnetic nanomaterials are good conductors, and a solid core made from such a material also constitutes a single short-circuited turn throughout its entire length. Eddy currents therefore circulate within the core in a plane normal to the flux, and are responsible for resistive heating of the core material. (2) Hysteresis losses: Each time the magnetic field is reversed, a small amount of energy is lost due to hysteresis within the core. For a given core material, the loss is proportional to the frequency, and is a function of the peak flux density to which it is subjected.

Ferromagnetic NPs possess hysteretic properties when exposed to alternating magnetic fields, which gives rise to magnetically induced heating. The amount of heat (power dissipation) generated per unit volume, $W$, is given by the frequency multiplied by the area of the hysteresis loop in the $\mathrm{M}-\mathrm{H}$ coordinates caused by hysteresis $\operatorname{loss}^{22}$ :

$$
W=-\mu_{0} \boldsymbol{f}_{\text {appl }} \oint H \cdot d M
$$

For ferromagnetic particles with sizes greater than those of superparamagnetic NPs, there is no explicit frequency dependence of the hysteresis loop area, so that the rate 
A

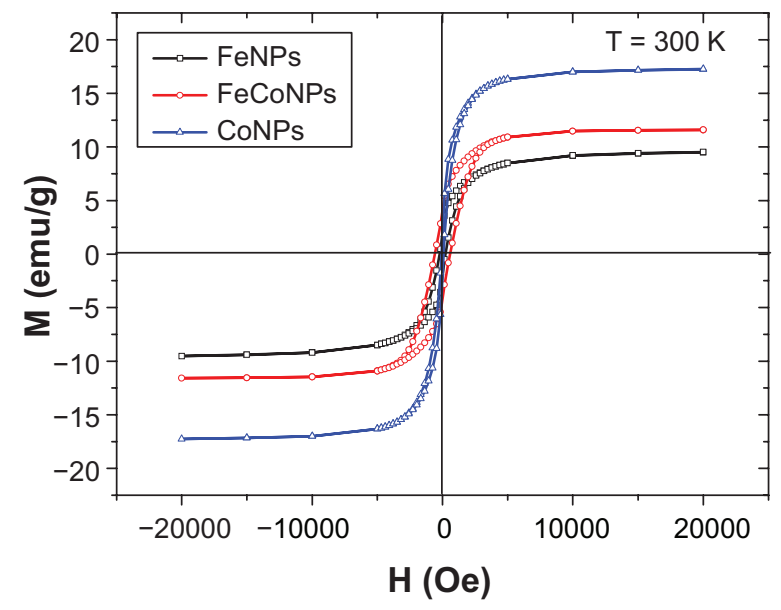

B

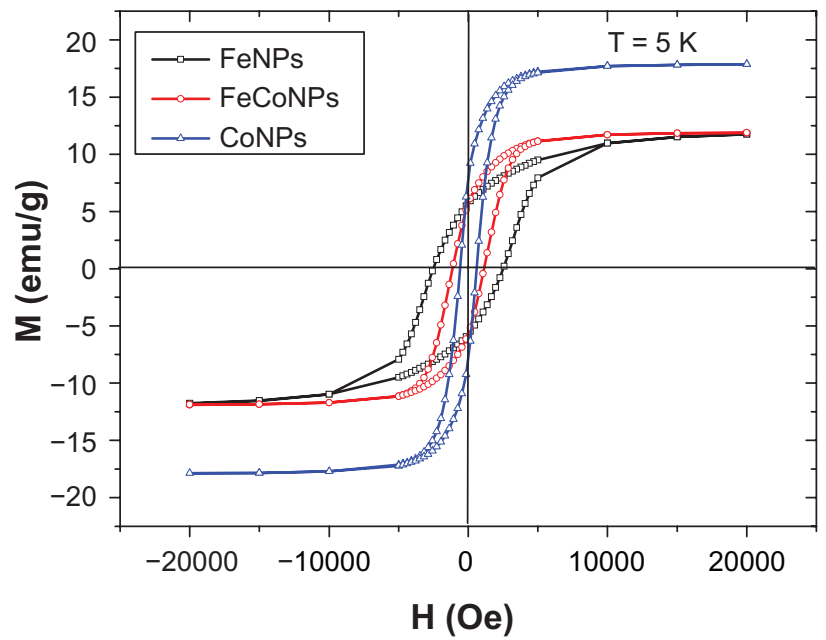

C

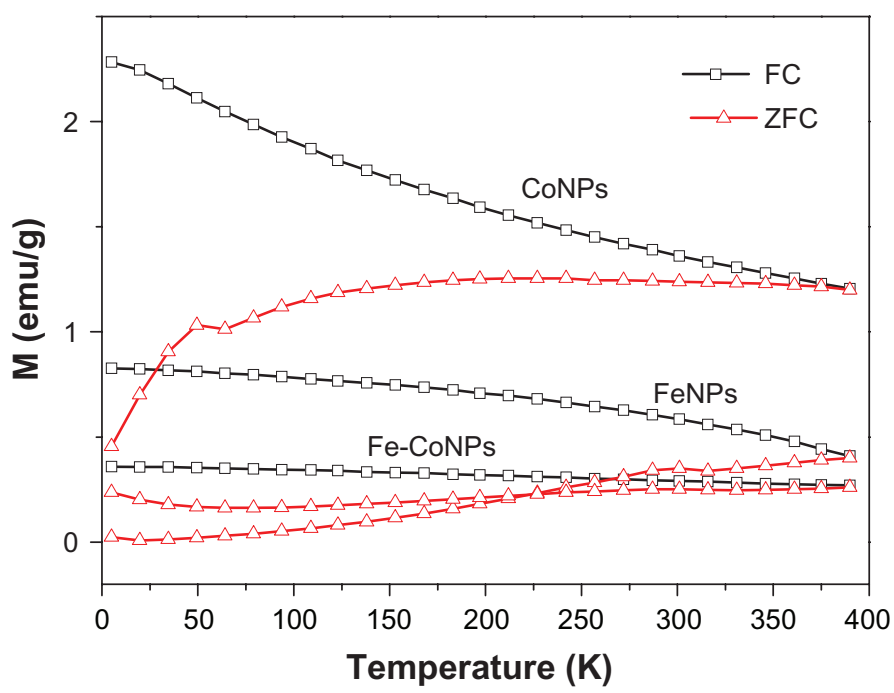

Figure 5 Magnetization curves of different magnetic NPs at $300 \mathrm{~K} \mathrm{~A}$ ) and $5 \mathrm{~K} \mathrm{~B}$ ); C) Temperature dependence of ZFC and FC measurements recorded at a magnetic field of 30 Oe for these magnetic NPs.

Abbreviations:

of heat emission can be determined from quasistatistical measurements of the hysteresis loop, for example, by means of SQUID magnetometery. Figure 5 compares magnetization loops measured at $5 \mathrm{~K}$ and $300 \mathrm{~K}$ for the $\mathrm{C}$ - $\mathrm{Fe}$, $\mathrm{C}-\mathrm{Fe} / \mathrm{Co}$, and C-Co NPs. The C-Co nanostructures revealed a larger saturation magnetic induction $\left(\mathrm{M}_{\mathrm{s}}\right)$, larger residual magnetization $\left(\mathrm{M}_{\mathrm{r}}\right)$, and smaller coercivity $\left(\mathrm{H}_{\mathrm{c}}\right)$ relative to the $\mathrm{C}-\mathrm{Fe} / \mathrm{Co}$ and $\mathrm{C}-\mathrm{Fe}$ nanostructures. One reason is the NP size distributions. The specific energy absorption multiplied by the particle density yields the thermal emission power in unit volume, $W$, which represents the efficiency of heating of the magnetic particles. This calculation allows NPs of various sizes to be compared as heat generators. The $\mathrm{Fe}$ and $\mathrm{Co}$ NPs in this work have sizes ranging from 7-15 nanometers.
For this size, the Fe NPs are about the size of the Fe domain $(14 \mathrm{~nm})$; however the Co NPs are much smaller than the Co domain size $(70 \mathrm{~nm}) .{ }^{23}$ Since the Fe NPs are composed most probably of single domains they exhibit the maximum possible coercivity for Fe, since the Co NPs which are much smaller compared to their domain size show a consequent diminished coercivity. This phenomenon is highlighted by the almost nonexistent hysteresis loss for the Co NPs. In the last decade, magnetic hyperthermia has been given a new impetus due to superparamagnetic liquids used as working agents. ${ }^{24,25}$ These are ferroliquids with one-domain magnetic NPs that are suspended in water and show superparamagnetic properties. When a ferroliquid is removed from the magnetic field, its magnetization vanishes because of the thermal energy of 
the environment. The other reason is that the greater pairing of electrons in Co and the stronger nuclear charge of the Co atoms lead to less surface delta multibonding of $\mathrm{Co}$ surface atoms in the Co NPs relative the Fe NPs. Such Co-C bonding lowers the coercivity of Co which results in far softer magnetic properties relative to Fe NPs. It is noteworthy that the magnetic hysteresis of Co NPs is almost zero at $300 \mathrm{~K}$, which indicates that the Co NPs are not agglomerated and are superparamagnetic. However, the Fe/Co and Fe NPs have more agglomerations, which were indicated in the larger hysteresis loops for these particles relative to Co NPs. As a result, $\mathrm{Fe}$ and $\mathrm{Fe} / \mathrm{Co}$ NPs can generate more thermal energy when they were exposure to the external AC field compared to the Co NPs.

A ferromagnetic particle becomes "superparamagnetic" below a critical size on the order of $100 \mathrm{~nm}$, depending on the material. Such a particle cannot develop internal magnetic domains and, therefore, acts as a paramagnetic particle with a magnetic moment of up to $10^{7}$ Bohr magnetons. The blocking phenomenon is a signature of the superparamagnetic regime that depends on the particle size, degree of crystallinity, and interparticle interactions. ${ }^{26}$ Each NP then becomes a single magnetic domain and shows superparamagnetic behavior when the temperature is above the so-called blocking temperature, $T_{B}{ }^{27}$ Below $T_{B}$, NPs are "blocked", which means that initially random magnetic moments of individual NPs cannot readily align with the applied field, because magnetic Zeeman $(H)$ and thermal fluctuation $\left(k_{B} T\right)$ energies are insufficient to overcome the energy barrier set by the magnetic anisotropy and interparticle dipolar interactions. Experimentally, $T_{B}$ is marked by the peak in the $M_{(T)}$ curve measured upon warming after a magnetic field is applied at a low temperature to a ZFC sample. It is well established that $T_{B}$ is reduced for smaller particles and for particles with reduced crystallinity; both properties lead to the reduction of the magnetic moment per particle. ${ }^{28}$ Another way to probe the magnetic response of a superparamagnetic assembly is to measure its magnetic moment at a fixed temperature as a function of the magnetic field. It is important to compare $M_{(H)}$ curves below and above $T_{B}$ to show the absence of the magnetic hysteresis above $T_{B}$. Figure $5 \mathrm{c}$ shows the results of ZFC measurements performed upon warming after a magnetic field of 30 Oe was applied at $5 \mathrm{~K}$ after cooling in zero applied fields. Clearly, Co NPs exhibit the lowest blocking temperature about $50 \mathrm{~K}$. Fe and Fe/Co NPs show an elevated $T_{B}$ of $300 \mathrm{~K}$. These results were fully consistent with the results of TEM; that is, Co NPs shown a relative smaller size and lower crystallinity than Fe and Fe/Co NPs.

\section{Conclusions}

This report demonstrated that $\mathrm{Fe}, \mathrm{Fe} / \mathrm{Co}$, and $\mathrm{Co}$ magnetic NPs with several graphitic layers were successfully synthesized. They were introduced to HeLa cervical cancer cell cultures and were found to be taken up by the cells and acted as high-efficiency RF absorbers. Under low frequency (350 kHz) RF radiation for a short exposure time of two minutes, the lowest concentration $(0.83 \mu \mathrm{g} / \mathrm{mL})$ of $\mathrm{C}-\mathrm{Fe}$ NPs was found to be the most suitable for inducing death in over $99 \%$ of the cancer cells. The RF-driven heating of the NPs is believed to be responsible for the generation of extremely localized damage inside and around the cells. The process described in this research has significant promise for various medical therapies and efficient ablation of tumors. A further continuation of this work involving the binding of these magnetic NPs to antibodies, proteins, and growth factors will lead to their specific delivery to the individual cancer cells, causing comparable thermal ablation of cancer cells while diminishing the potential toxicity of these entities to normal cells. This research should also advance towards finding more RF-sensitive nanomaterials materials that are able to provide thermal ablation of cancer cells, but in very low concentrations that avoid the possible cytotoxic and undesirable effects that these materials could induce in healthy tissues.

\section{Acknowledgments}

This research was financial support from Arkansas Science and Technology Authority (ASTA) grant \# 08-CAT-03 is highly appreciated. Technical support from Carl J Plumley is also acknowledged. The authors report no conflicts of interest in this work.

\section{References}

1. Douziech-Eryrolles L, Marchais H, Herve K, et al. Nanovectors for anticancer agents based on superparamagnetic iron oxide nanoparticles. Int J Nanomedicine. 2007;2:541-550.

2. Reiss G, Huetten A. Magnetic nanoparticles: applications beyond data storage. Nat Mater. 2005;4:725-726.

3. Bulte JWM, Kraitchman DL. Iron oxide MR contrast agents for molecular and cellular imaging. NMR Biomed. 2004;17:484-499.

4. Corot C, Port M, Guilbert I, et al. Superparamagnetic Contrast Agents Boca Raton, FL: CRC Press LLC; 2007:59-83.

5. Lu AH, Schmidt W, Matoussevitch N, et al. Nanoengineering of a magnetically separable hydrogenation catalyst. Angew Chem. 2004;116:4403-4410.

6. Hyeon T. Chemical synthesis of magnetic nanoparticles. Chem Commun. 2003;8:927-934

7. Yavuz CT, Mayo JT, Yu WW, et al. Low-field magnetic separation of monodisperse Fe3O4 nanocrystals. Science. 2006;314:964-967.

8. Seo WS, Lee JH, Sun X, et al. FeCo/graphitic-shell nanocrystals as advanced magnetic resonance imaging and near-infrared agents. Nat Mater. 2006;5:971-976. 
9. Wang Y, Wei W, Maspoch D, Wu J, Dravid VP, Mirkin CA. Superparamagnetic sub-5 nm Fe@C nanoparticles: isolation, structure, magnetic properties, and directed assembly. Nano Lett. 2008;8: 3761-3765.

10. Xu Y, Mahmood M, Li Z, et al. Cobalt nanoparticles coated with graphitic shells as localized radio frequency absorbers for cancer therapy. Nanotechnology. 2008;19:435102-425108.

11. Lu A, Salabas EL, Schuth F. Magnetic nanoparticles: synthesis, protection, functionalization, and application. Angew Chem Int Ed Engl. 2007;46:1222-1225.

12. Geng J, Jefferson DA, Johnson BFG. Direct conversion of iron stearate into magnetic $\mathrm{Fe}$ and $\mathrm{Fe} 3 \mathrm{C}$ nanocrystals encapsulated in polyhedral graphite cages. Chem Comm. 2004;(21):2442-2443.

13. Vilas GP, Swati VP, Aharon G. Semiconducting, magnetic or superconducting nanoparticles encapsulated in carbon shells by RAPET method. Carbon Sci Tech. 2008;1:46-56.

14. Hu L, Chen J, Liu W, Xiie Q, Kajdas C. Investigations of tribochemical behaviour of Al-Si alloy against itself lubricated by anines. Wear. 2000;243:60-67.

15. McIntyre NS, Cook MG. X-Ray photoelectron studies on some oxides and hydroxides of cobalt, nickel, and copper. Anal Chem. 1975;47: 2208-2213.

16. Briggs D, Seah MP. Practical surface analysis by auger and X-ray photoelectron spectroscopy. 2nd Ed. Chichester, UK: J Wiley \& Sons; 1999.

17. Leea $\mathrm{S}$, Johnson $\mathrm{D}$, Dunbar $\mathrm{K}$, et al. $2.45 \mathrm{GHz}$ radiofrequency fields alter gene expression in cultured human cells. FEBS Letters. 2005;579:4829-4836.

18. Young JH, Wang MT, Brezovich IA. Frequency/depth-penetration considerations in hyperthermia by magnetically induced currents. Electron Lett. 1980;16:358-359.
19. Roschmann P. Radiofrequency penetration and absorption in the human body: limitations to high field whole body nuclear magnetic resonance imaging. Med Phys. 1987;14:922-931.

20. Nikiforov VN. Magnetic induction hyperthemia. Russian Physics Journal. 2007;50:913-924.

21. Kan S, Sachan M, Kirchhoff J, Majetich SA. Crystallographic alignment of nanoparticles during self assembly. IEEE Trans Magn. $2005 ; 41-45$.

22. Rosensweig RE. Heating magnetic fluid with alternating magnetic field. J Magn Magn Mater. 2002;252:370-374.

23. Zhao L, Zhang H, Xing Y, et al. Studies on the magnetism of cobalt ferrite nanocrystals synthesized by hydrothermal method. J Solid State Chem. 2008;181:245-252.

24. Jordan A, Scholz R, Wust P. Magnetic fluid hyperthermia (MFH): Cancer treatment with AC magnetic field induced excitation of biocompatible superparamagnetic nanoparticles. J Magn Magn Mater. 1999;201:413-419.

25. Jordan A, Scholz R, Maier-Hauff K, et al. Presentation of a new magnetic field therapy system for the treatment of human solid tumors with magnetic fluid hyperthermia. J Magn Magn Mater. 2001;225:118-125.

26. Prozorov R, Yeshurun Y, Prozorov T, Gedanken A. Magnetic irreversibility and relaxation in assembly of ferromagnetic nanoparticles. Phys Rev B Condens Matter. 1999;59:6956-6965.

27. Prozorov T, Palo P, Wang L, et al. Cobalt ferrite nanocrystals: outperforming magnetotactic bacteria. ACS Nano. 2007;1:228-233.

28. Prozorov R, Prozorov T. Effective collective barrier for magnetic relaxation in frozen ferrofluids. J Magn Magn Mater. 2004;281:312-317.
International Journal of Nanomedicine

\section{Publish your work in this journal}

The International Journal of Nanomedicine is an international, peerreviewed journal focusing on the application of nanotechnology in diagnostics, therapeutics, and drug delivery systems throughout the biomedical field. This journal is indexed on PubMed Central, MedLine, CAS, SciSearch $\AA$, Current Contents ${ }^{\circledR} /$ Clinical Medicine,

\section{Dovepress}

Journal Citation Reports/Science Edition, EMBase, Scopus and the Elsevier Bibliographic databases. The manuscript management system is completely online and includes a very quick and fair peer-review system, which is all easy to use. Visit http://www.dovepress.com/ testimonials.php to read real quotes from published authors. 